\title{
TRATAMENTO DOS MEDULOBLASTOMAS DO CEREBELO
}

\author{
Walter C. Pereira * \\ JULINHO AISEN *** \\ Vivaldino P. Franciosi \\ Rolando A. Tenuto ** \\ JosÉ LUZIo *
}

A conduta terapêutica nos meduloblastomas do cerebelo tem sido muito discutida. Cushing (citado por Smith e col. ${ }^{10}$ ), em 1903, pela primeira vez atuou cirùrgicamente sôbre esta neoplasia, praticando exérese parcial por aspiração. Este mesmo autor em 1919 iniciou radioterapia dirigida à fossa posterior e, em 1927, também ao longo da coluna vertebral, como complementação da cirurgia. Desde então a maioria dos autores segue esta orientação', :a, 4, 5, 6, 7, 10, 11. Inicialmente é feita craniectomia da fossa posterior para, mediante remoção parcial ou subtotal do tumor, desobstruir as vias do líquido cefalorraqueano (LCR) e, a seguir, radioterapia sôbre a fossa posterior e ao longo da coluna vertebral afim de destruir eventuais metástases.

Cutler e col. ${ }^{3}$, em 1936, em virtude da elevada mortalidade operatória nas intervenções sôbre meduloblastomas do cerebelo (em tôrno de 25\% segundo a maioria dos autores), preferem fazer irradiação do tumor sem prévia descompressão cirúrgica da fossa posterior. Para reduzir a hipertensão intracraniana preconizam o uso de desidratantes no início da radioterapia. Registram, com esta conduta, melhores resultados que os obtidos na intervenção direta sôbre a neoplasia. Consideram a resposta à radioterapia um teste tão valioso como o próprio exame histopatológico para a confirmação diagnóstica do meduloblastoma. Apontam, ainda, a manipulação cirúrgica do tumor como favorecedora da disseminação de metástases pelo espaço subaracnóideo.

Peirce e col. ${ }^{8}$, em 1949, recomendam biopsia antes da irradiação. A colheita do material para exame histopatológico é feita mediante perfuração occipital, sendo o tumor aspirado por cânula especial. Evitam, dessa maneira, que neoplasisas benignas, como astrocitomas, possam ser confundidas com o meduloblastoma e assim protelado seu tratamento radical pela cirurgia. Julgam desnecessária a descompressão da fossa posterior antes da radioterapia, praticando, quando necessário, punções ventriculares para aliviar a hipertensão intracraniana.

Trabalho da Clinica Neurológica (Prof. Adherbal Tolosa) e do Serviço de Radioterapia (Prof. Antonio Costa Pinto) da Faculdade de Medicina da Universidade de São Paulo, apresentado no vI Congresso da Sociedade Brasileira de Neurocirurgia (São Paulo, 1966): * neurocirurglões; ** neurocirurgião-chefe; *** residente de neurocirurgia; **** médico auxiliar do Serviço de Radioterapia. 
Tendo em vista a divergência de opiniões quanto à conduta e resultados obtidos no tratamento de meduloblastomas do cerebelo, propomo-nos, neste trabalho, a analisar material acumulado nos últimos dez anos.

\section{MATERIAL}

Foram estudados 60 casos de pacientes com diagnóstico clínico-iodoventriculográfico de neoplasia do cerebelo, operados entre os anos de 1956 e 1965, sendo 35 do sexo masculino (58\%) e 25 do feminino (42\%). As Idades variaram de 1 a 56 anos, estando $75 \%$ dos pacientes dentro da primeira década da vida (quadro 1). Os sintomas evoluiram de 1 até 24 meses, tendo em $76 \%$ dos casos duração inferior ou igual a 6 meses (quadro 2). Todos os doentes apresentavam sintomas e/ou sinais

\begin{tabular}{cc}
\hline $\begin{array}{l}\text { IDADE } \\
\text { (anos) }\end{array}$ & $\begin{array}{l}\text { N. } D E \\
C A S O S\end{array}$ \\
\hline $1-5$ & 18 \\
$6-10$ & 27 \\
$11-15$ & 11 \\
25 & 1 \\
30 & 1 \\
50 & 2 \\
\hline
\end{tabular}

Quadro 1 - Distribuição etária de 60 pacientes com meduloblastoma do cerebelo.

\begin{tabular}{cc}
\hline $\begin{array}{c}\text { DURACAO DOS SINTOMAS } \\
\text { (meses) }\end{array}$ & $\begin{array}{c}\text { N.P DE } \\
\text { CASOS }\end{array}$ \\
\hline $1-3$ & 28 \\
$4-6$ & 18 \\
$7-9$ & 6 \\
$10-12$ & 2 \\
$13-24$ & 6 \\
\hline
\end{tabular}

Quadro 2 - Duração dos sintomas em 60 pacientes com meduloblastoma do cerebelo.

de hipertensão intracraniana, sendo o papiledema encontrado em $90 \%$ e atrona secundáría das papilas ópticas em $3 \%$ dos casos. Sinais de comprometimento vermiano foram observados em $80 \%$ dos pacientes, 1soladamente ou associados a sinais cerebelares apendiculares em $52 \%$ dos casos. Sinats de lesăo piramidal, uni ou 
bilateral, também foram frequientemente observados (42\%), sendo mais raro o comprometimento de nervos cranianos (17\%).

A maioria dos pacientes fol submetida aos exames complementares de rotina, tais como cranlograma, eletrencefalograma e análise do LCR obtido, em geral, por punçāo ventricular. A carótido-anglografia fol feita na quase totalidade dos casos e a lodoventriculografia em todos.

Quanto à conduta terapêtica os pacientes foram divididos em dois grupos:

GRUPO A - Em 16 pacientes fol feita inicialmente, craniectomia da fossa posterior, com exérese parcial ou subtotal da neoplasia, seguida de cobaltoterapia nos pacientes que sobreviveram ao pós-operatório imediato. Essa conduta foi motivada por um ou mais dos seguintes fatôres: a) em alguns casos a idade dos pacientes era aparentemente incompativel com o diagnóstico de meduloblastoma (dois com mais de 50, um com 30 e outro com 25 anos), tendo o exame histopatológico confirmado, ulteriormente, tal diagnóstico; b) presença de sinais cerebelares predominantemente apendiculares; c) lodoventriculografia demonstrando desvios laterais do 4.9 ventriculo e/ou do aqueduto cerebral, sugestivos de tumores dos hemisférios cerebelares.

Os resultados obtidos neste grupo, quer no que se refere à ocorrencla de complicações pós-operatórias, quer referentes ao número de óbitos, precoces ou tardios, foram bastante precários (quadro 3).

GRUPO B - Em 44 pacientes fol adotada a seguinte conduta: intervenção cirúrgica para derivar o trânsito do LCR seguida de cobaltoterapia sôbre a fossa posterior e ao longo da coluna vertebral. Esta orientaça foi baseada no diagnóstico clinico-iodoventriculografico de meduloblastoma do cerebelo, que tem como elementos fundamentais os seguintes fatos: a) idade dos pacientes inferior ou igual a

\section{$\frac{\text { Complicaçóes }}{\text { HIC Fist. Mening. }} \frac{\text { Obitos }}{\text { OP-PO 1-3m }} \quad$ Evolução favorável}

\begin{tabular}{ccccccccc}
$\begin{array}{c}\text { Número } \\
\text { de } \\
\text { casos }\end{array}$ & 11 & 4 & 4 & 9 & 4 & 3 casos seguidos durante \\
\cline { 2 - 3 } & $81 \%$ & & & $81 \%$ & & 14,16 e 36 meses
\end{tabular}

Quadro 3 - Resultados da exerese parcial do tumor e cobaltoterapia em 16 pacientes com meduloblastoma do cerebelo. Abreviaturas: Fist. = fistula de LCR; HIC = hipertensão intracraniana; Mening. $=$ meningite; $m=$ mês ou meses; oP $=$ operatorio; $P O=$ pos-operatório.

\begin{tabular}{|c|c|c|c|c|c|c|c|c|c|}
\hline & \multicolumn{3}{|c|}{$\begin{array}{c}\text { Tipo de } \\
\text { derivacão }\end{array}$} & \multicolumn{3}{|c|}{ Complicaçoes } & \multicolumn{2}{|c|}{ Obitos } & \multirow{2}{*}{$\begin{array}{l}\text { Evolução } \\
\text { favorável }\end{array}$} \\
\hline & $L T$ & Laz. & $V A$ & $H I C$ & Fist. & Mening. & $O P-P O$ & Tardio & \\
\hline \multirow{2}{*}{$\begin{array}{c}\text { Número } \\
\text { de } \\
\text { casos }\end{array}$} & 38 & 4 & 2 & 12 & 1 & 1 & 3 & 13 & \multirow[t]{2}{*}{$(63 \%)$} \\
\hline & & & & \multicolumn{3}{|c|}{$32,5 \%$} & \multicolumn{2}{|c|}{$37 \%$} & \\
\hline
\end{tabular}

Quadro 4 - Resultados do tratamento de 44 pacientes com diagnóstioo clinico-iodoventriculográfico de meduloblastoma do cerebelo, submetidos a derivacões do LCR e cobaltoterapia. Abreviaturas: Fist. = fistula de LCR; HIC = hipertensão intracraniana; Laz. = operacão de Lazorthes; LT = abertura da lamina terminal; Mening. $=$ meningite; $O P=$ operatoria; $P O=$ pós-operatório;

$V A=$ ventrículo-auriculostomia. 
10 anos; b) início da sintomatologia inferior a 6 meses; c) predominância nitida de comprometimento cerebelar vermiano; d) ausêneia de desvios laterais consideráveis do 4.9 ventrículo e/ou do aqueduto cerebral na iodoventriculografia.

A cobaltoterapia é iniciada de 6 a 10 dias depois da derivação do trânsito do LCR. Se após 8 a 10 aplicações os sintomas neurológicos permanecerem inalterados, ou se agravarem, está indicada a exploração cirúrgica da fossa posterior. E preciso cautela, contudo, para não confundir a melhora do paciente pelo alívio da hipertensão intracraniana, que se processa logo após a derivação do trânsito do LCR, com a regressão dos sintomas cerebelares ou decorrentes da compressão do tronco cerebral pela neoplasia.

A exploração cirúrgica da fossa posterior foi feita em 9 doentes dêste grupo, tendo em 6 confirmado a presença de meduloblastoma e, em 3 , demonstrado tratar-se de astrocitomas cerebelares. Dêstes 9 pacientes, 7 faleceram no pós-operatório imediato; entre êstes estão incluídos dois casos de astrocitoma. O tempo decorrido entre a intervenção para a derivação do trânsito do LCR e a crantectomia da lossa posterior variou de 18 dias até 2 anos. Na maioria das vêzes a segunda intervenção foi realizada por ter havido recidiva da sintomatologia após períodos variáveis de tempo em que se observou melhora acentuada ou normalização do exame neurológico.

Nas primeiras aplicações de cobaltoterapia costuma haver agravamento transitório dos sintomas neurológicos determinado, provàvelmente, por edema encefálico. No entanto, êstes sintomas regridem ràpidamente e o paciente, depois de 8 a 10 aplicações, já deve apresentar nítida melhora do quadro inicial se o dlagnóstico. de meduloblastoma estiver correto.

\begin{tabular}{|c|c|}
\hline $3-6$ meses & 8 casos \\
\hline 6 meses -1 ano & 2 casos \\
\hline $1-2$ anos & 2 casos \\
\hline $2-3$ anos & 1 caso \\
\hline $\begin{array}{l}\text { Quadro } 5 \text { - Temp } \\
\text { pacientes com dia } \\
\text { culografico de me } \\
\text { submetidos a deriv }\end{array}$ & $\begin{array}{c}13 \text { dos } 44 \\
\text { doventri- } \\
\text { cerebelo, } \\
\text { colbalto- }\end{array}$ \\
\hline 6 meses -1 ano & 13 casos \\
\hline $1-2$ anos & 6 casos \\
\hline $2-3$ anos & 3 casos \\
\hline $3-4$ anos & 2 casos \\
\hline $4-5$ anos & 1 caso \\
\hline $5-6$ anos & 1 caso \\
\hline $6-7$ anos & 0 \\
\hline $7-8$ anos & 2 casos \\
\hline
\end{tabular}

Quadro 6 - Tempo de seguimento de 28 dos 44 pacientes com diagnóstico clinico-iodoventriculográfico de meduloblastoma do cerebelo, submetidos a derivaçóes do LCR e cobaltoterapia. 
Os resultados obtidos com êste tipo de tratamento foram consideràvelmente melhores do que aquêles verificados no grupo $A$, quer no que se refere à incidência de complicações pós-operatórias, quer referentes ao número de óbitos precoces ou tardios (quadro 4).

Dos 16 óbitos (37\%) ocorridos neste grupo, 7 se verificaram no pós-operatório imediato de exploraçōes da fossa posterior; este fato confirma a elevada incidência de mortalidade na cirurgia direta sôbre o meduloblastoma do cerebelo, também observada no grupo A. Entre os 16 pacientes que faleceram, três sobreviveram mais de um ano (quadro 5 ).

Dos 28 casos com evolução favorável, 15 foram acompanhados durante períodos superiores a um ano, com melhora acentuada ou normalização do exame neurológico. Entre êstes últimos há três pacientes com seguimento superior a 5 anos, que levam vida inteiramente normal (quadro 6).

Em 15, dos 44 pacientes dêste grupo, a neoplasia fol examinada histologica. mente (casos de necropsia e/ou de exploração cirúrgica). Em 11 casos fol conlirmando o diagnóstico de meduloblastoma $(73 \%)$ e, em 4, foram encontrados outros tipos de tumor: três astrocitomas e um cisto dermóide do cerebelo. Em nenhum dos casos de necropsia foram encontradas lesões do parênquima encefálico atribulveis à açāo da cobaltoterapia. As neoplasias, ao contrário, apresentavam sempre grau intenso de necrose e fibrose secundárias. Em 3 pacientes foram verificadas metastases medulares e supratentoriais difusas.

A cobaltoterapia foi empregada em ambos os grupos. A dose total média pór série foi de $3.000 \mathrm{rds}$ na fossa posterior e de $2.000 \mathrm{rds}$ ao longo da coluna vertebral. Esta dose é fracionada em doses diárias de 100 a 200 rds. O número de séries aplicadas variou de 1 a 5 (em apenas um caso), sendo na maioria das vêzes feitas duas séries. A segunda série foi feita de rotina, em geral 6 meses após a primeira, mesmo nos pacientes sem recidiva clínica da neoplasia. Outras séries foram indicadas nos casos com recidiva dos sintomas neurológicos após períodos variáveis de tempo. Em alguns doentes esta repetição fol precedida de nova iodoventriculografia ou de carótido-angiografia para a verificaçāo de possíveis metástases supratentoriais. Em três pacientes foi observada boa resposta à primeira série de radioterapia, ocorrendo, porém, recidiva dos sintomas neurológicos após períodos de 6 meses a um ano. Submetidos à nova série de cobaltoterapia nắo foi obtiđa resposta satisfatória desta vez, sendo então explorados cirùrgicamente. Em todos foram encontradas neoplasias, histologicamente diagnosticadas como meduloblastoma, com grau acentuado de necrose e metástases medulares e supratentoriais.

Além da alopecia, sempre observada, a única complicação atribuivel à cobaltoterapia foi plaqueto-e leucopenia, verificadas em apenas um caso. Esta paciente estava sendo tratada com antibióticos e quimioterápicos, que também podem ser responsabilizados pelo quadro de grave depressão medular encontrado á necropsia.

\section{COMENTARIOS}

Os resultados do tratamento do meduloblastoma do cerebelo são precários. Tratando-se de neoplasia extremamente maligna, com caráter invasivo e fácil disseminação de metástases pelo LCR, sua erradicação pela cirurgia é impraticável ${ }^{5}, 9$. A radioterapia, complementando a exérese parcial do tumor, melhorou sensivelmente a sobrevida de pacientes com meduloblastoma, havendo casos referidos na literatura com mais de 5 anos de sesuimento $1,6,7,10,11$. A mortalidade operatória é, contudo, muito elevada nas intervenções diretas sôbre a neoplassia referindo, a maioria dos autores, cêrca de $25 \%$ de óbitos intra-operatórios ou imediatamente após a cirurgia. Por êste motivo, e pela impossibilidade de remoção completa, preferem alguns cirurgiões fazer sòmente biopsia mediante trépano-punção ${ }^{8}$, 
ou irradiar a fossa posterior sem qualquer intervenção neurocirúrgica prévia, baseando o diagnóstico exclusivamente no quadro clínico ${ }^{3}$.

Em 16 pacientes (grupo A), de um total de 60 com diagnóstico clínico-iodoventriculográfico de tumor do cerebelo, foi feita inicialmente craniectomia da fossa posterior e exérese parcial ou subtotal da neoplasia. Em todos êstes casos o exame histológico demonstrou meduloblastomas. Após a intervenção cirúrgica foi feita cobaltoterapia sôbre a fossa posterior e ao longo da coluna vertebral, nos pacientes que sobreviveram ao pós-operatório imediato. A mortalidade intra e pós-operatória imediata atingiu a $56 \%$ e nos seguintes 3 meses a $81 \%$ dos casos. O número de complicações foi também muito elevado $(81 \%)$. Sòmente três pacientes (19\%) sobreviveram mais de um ano (quadro 3 ).

Em 44 pacientes (grupo B) suspeitou-se de meduloblastoma do cerebelo pelos quadros clínico e iodoventriculográfico. Neste grupo foi feita derivação do trânsito do LCR e, a seguir, cobaltoterapia sôbre a fossa posterior e coluna vertebral. As complicaçōes pós-operatórias, assim como a mortalidade precoce e tardia, foram consideràvelmente menos numerosas do que no grupo A (quadros 4 e 5). Cêrca de $40 \%$ dos pacientes sobreviveram mais de um ano após o início do tratamento, sendo que três estão sendo acompanhados há mais de 5 anos (quadro 6). Em apenas 4, de 15 casos estudados histologgicamente, houve discordância entre êste exame e o diagnóstico clínico-iodoventriculográfico $(27 \%)$ : três astrocitomas e um cisto dermóide do cerebelo.

Em nossa experiência, portanto, a derivação do trânsito do LCR seguida de cobaltoterapia sôbre a fossa posterior e ao longo da coluna vertebral, proporcionou resultados nitidamente melhores do que a intervenção cirúrgica direta sôbre o meduloblastoma complementada com cobaltoterapia. Dos 4 pacientes em que o diagnóstico clínico-iodoventriculográfico de meduloblastoma do cerebelo não foi confirmado pelo exame histológico, três foram ulteriormente, submetidos à craniectomia da fossa posterior, pois não houve resposta satisfatória à cobaltoterapia. Em apenas um caso o êrro diagnóstico foi verificado na mesa de necropsia, porque a exploração cirúrgica da neoplasia não fôra possivel em virtude das péssimas condições gerais em que a paciente se encontrava.

Julgamos que as possíveis críticas relativas à perda de tempo em se irradiar neoplasias não sensiveis à cobaltoterapia são improcedentes, porquanto o atrazo a que tais casos são sujeitos não deve ir além de 15 a 20 dias, segundo a conduta que adotamos. Tal atrazo não significa grande prejuizo para o paciente, uma vez que a hipertensão intracraniana, que constitui o principal fator de agravamento do quadro, fica controlada com a derivação do trânsito do LCR. Além disso, as neoplasias não radiossensiveis, são, comumente, de crescimento lento.

Não obstante a falta de rigor científico na comprovação diagnóstica da maioria de nossos casos, julgamos que os resultados obtidos justificam plenamente a conduta que, em nossa experiência, possibilitou maior sobrevida média, em se tratando de afecção de sombrio prognóstico. 


\section{RESUMO}

São analisados 60 casos de pacientes com diagnóstico clínico e iodoventriculográfico de meduloblastoma do cerebelo. Em 16 casos foi feita craniectomia da fossa posterior, para exérese parcial da neoplasia, seguida de cobaltoterapia. Em 44 pacientes foi feita derivação do trânsito do líquido cefalorraqueano e, a seguir, cobaltoterapia. Os resultados obtidos foram nitidamente melhores no grupo em que não houve intervenção direta sôbre a neoplasia.

\section{SUMMARY}

Medulloblastoma of the cerebellum: an analysis of the therapeutic results

Sixty patients operated on with a diagnosis of medulloblastoma of the cerebellum based on clinical and iodized oil ventriculography data are analysed. In 16 cases posterior fossa craniectomy was performed for partial remotion of the growth, followed by cobaltotherapy. In 44, after a shunt procedure cobaltotherapy was started. The results were definitely better in the group without direct manipulation of the tumor.

\section{REFERENCIAS}

1. BERGER, E. C. \& ELVIDGE, A. R. - Medulloblastomas and cerebellar sarsomas. A clinical survey. J. Neurosurg. 20:139-144, 1963. 2. CUSHING, H. - Experiences with cerebellar medulloblastomas: critical review. Acta path. microbiol. scand, 7:1-86, 1930. 3. CUTLER, E. C.; SOSMAN, M. C. \& VAUGHAN, W. W. The place of irradiation in the treatment of cerebellar medulloblastomata. Report of 20 cases. Amer. J. Roentgenol. 35:429-450, 1963. 4. DYKE, C. \& DAVIDOFF, L. - Roentgen Treatment of Diseases of the Nervous System. Lea \& Febiger, Filadelfia, 1942. 5. INGRAHAM, F. D. \& MATSON, D. D. - Neurosurgery of Infancy and Childhood. Charles Thomas, Springfield, 1954. 6. LAMPE, J. \& Mac INTYRE, R. S. - Medulloblastoma of the cerebellum. Arch. Neurol. Psychiat. 62: 322-329, 1949. 7. LAMPE, J. \& Mac INTYRE, R. S. - Experiences in the radiation therapy of medulloplastoma of the cerebellum. Amer. J. Roentgenol. 71:659-668, 1954. 8. PEIRCE, C. B.; CONE, W. V.; BOUCHARD, J. \& LEWIS, R. C. - Medulloblastoma: non-operative management with roentgentherapy after aspiration biopsy. Radiology. 52:621-632, 1949. 9. RUSSELL, D. S. \& RUBINSTEIN, L. J. - The Pathology of Tumours of the Nervous System. Edward Arnold, Londres, 1959.10. SMITH, R. A.; LAMPE, J. \& KAHN, E. A. - The prognosis of medulloblastoma in children. J. Neurosurg. 18:91-97, 1961. 11. SPITZ, E. B.; SHENKIN, H. A. \& GRANT, F. C. - Cerebellar medulloblastoma in adults. Arch. Neurol. Psychiat. 57: 417-422, 1947 .

Clinica Neurológica - Hospitat das Clinicas da Faculdade de Medicina da Universidade de São Paulo — Caixa Postal \$461 - São Paulo, SP - Brasil. 